\title{
AVALIAÇÃO DE DANOS POR INSETOS EM TORAS ESTOCADAS EM INDÚSTRIAS MADEIREIRAS DE MANAUS, AMAZONAS, BRASIL ${ }^{1}$
}

\author{
Raimunda Liége Souza de Abreu², Ceci Sales-Campos², Rogério Eiji Hanada², Francisco José de Vasconcellos ${ }^{2}$ e
} Jorge Alves de Freitas ${ }^{2}$

\begin{abstract}
RESUMO - Em seis indústrias madeireiras de Manaus, Amazonas, foi realizado um trabalho de pesquisa, com a utilização de um questionário, para averiguar as condições de uso e processamento da madeira e as medidas preventivas contra o ataque de insetos. Foram realizados, também, um levantamento da ocorrência de insetos em 19 espécies de madeiras utilizadas por essas indústrias e a avaliação do dano provocado pelas principais espécies de Coleoptera (besouros) e Isoptera (cupins). Das respostas apuradas, constatou-se que nenhuma das empresas visitadas emprega qualquer produto para prevenir o ataque de insetos às toras, assim como a secagem e a estocagem das toras são feitas de forma incorreta, contribuindo para aumentar a intensidade de ataque de insetos. Foram encontradas uma família de cupins e 16 de besouros, ressaltando que destas apenas cinco causam danos à madeira. Do total de 13 espécies de insetos coletados, destacam-se Xyleborus affinis Eichhoff e Platypus parallelus (Fabricius), encontradas em 18 espécies madeireiras, sendo conseqüentemente responsáveis pela maioria dos danos nas toras; $X$. volvulus (Fabricius) e Platypus sp. foram encontradas em cinco espécies; X. ferrugineus (Fabricius) em três espécies; Minthea rugicolis Walk, Minthea sp. e Nasutitermes corniger (Motschulsky) em duas, e Dinoderus bifoveolatus Wollaston, Anoplotermes sp.; e Cnesinus sp. em uma. As espécies de madeiras que sofreram maior grau de deterioração, causada principalmente por coleópteros, foram Ceiba pentandra (L.) Gaertn. e Copaifera multijuga Hayne, seguidas por Couroupita guianensis Aubl., Calophyllum brasiliense Cambess., Cedrela odorata L., Hevea brasiliensis Müll. Arg., Hura crepitans L., Hymenolobium sp., Maquira coriacea (Karsten) C.C. Berg, Nectandra sp., Virola surinamensis Warb. e Vochysia sp.
\end{abstract}

Palavras-chaves: Madeira, besouro, cupim, levantamento e deterioração de madeira.

\section{EVALUATION OF INSECT DAMAGE TO STORED LOGS IN SIX WOOD INDUSTRIES IN MANAUS, AMAZONAS}

\begin{abstract}
This work was carried out in two stages in six wood industries of Manaus, Amazonas. In the first stage, the handling conditions, wood processing as well as the preventive measures against insect attack were evaluated through a questionnaire. In the second stage an evaluation was conducted on the incidence of insects in 19 forest species utilized by those industries and on the deterioration degree caused by the main species of Coleoptera (beetles) and Isoptera (termites). One family of termites and five to 16 families of beetles were found to cause damage on wood. Out of the thirteen beetles collected, Xyleborus affinis Eichhoff and Platypus parallelus (Fabricius) stand out and were found in 18 wood species. Consequently, they were found to be responsible for most of the wood damages. X. volvulus (Fabricius) and Platypus sp. were found in five species, $\boldsymbol{X}$. ferrugineus (Fabricius) in three species, Minthea rugicolis Walk, Minthea sp. and Nasutitermes corniger (Motschulsky) in two, and Anoplotermes sp. in one species. The wood species, Ceiba pentandra (L.) Gaertn. and Copaifera multijuga Hayne presented the highest degree of deterioration, caused mainly by Coleoptera, followed by Couroupita guianensis Aubl., Calophyllum brasiliense Cambess., Cedrela odorata L., Hevea brasiliensis Mull. Arg., Hura crepitans L., Hymenolobium sp., Maquira coriacea (Karsten) C.C. Berg, Nectandra sp., Virola surinamensis Warb. and Vochysia sp.
\end{abstract}

Key words: Beetle, termite, survey, wood deterioration.

1 Recebido para publicação em 11.6.2001.

Aceito para publicação em 3.12.2002.

2 Pesquisadores do Instituto Nacional de Pesquisas da Amazônia, Coordenação de Pesquisas em Produtos Florestais, Av. André Araújo, 2936, Caixa Postal 478, Petrópolis, 69011-970, Manaus-AM, Brasil, <raiabreu@inpa.gov.br>, <ceci@inpa.gv.br>, <rhanada@inpa.gov.br>, <chicao@inpa.gov.br>, <jorginho@inpa.gov.br>. 
Na Região Amazônica, apesar de existir um grande potencial madeireiro, o setor que explora este ramo de atividade vem enfrentando uma série de dificuldades no que tange ao ataque de insetos, principalmente por causa de suas consequiências, uma vez que medidas preventivas e curativas são raras.

As perdas em termos de volume de tora em algumas indústrias madeireiras chegam a $60 \%$, principalmente por causa da falta de cuidados com a matéria-prima no pósabate, transporte e armazenamento. Como as árvores são geralmente derrubadas na época da vazante e as toras permanecem no piso da floresta, aguardando a elevação das águas para serem transportadas, tornam-se suscetíveis ao ataque de insetos e outros organismos deterioradores de madeira. Quando as toras chegam ao pátio das indústrias, parte delas já está bastante deteriorada em virtude do ataque desses organismos (Sales-Campos et al., 2000).

É do conhecimento que, dentre os cupins e besouros que habitam a madeira, existem aqueles que atacam a madeira com altos teores de umidade e aqueles que preferem a madeira seca. Dentre as famílias de besouros que atacam árvores recém-abatidas podem-se destacar Scolytidae e Platypodidae e algumas espécies de Cerambycidade e Curculionidae. Dos besouros que atacam madeira seca, preferindo umidade abaixo de 50\%, destacam-se Lyctidae, Bostrichidae e Anobiidae (GRAY, 1972). Dentre os cupins destacam-se as famílias Termitidae e Rhinotermitidae, com hábitos variados, os quais podem ser xilófagos, fitófagos e também podem alimentar-se de húmus (Bandeira, 1989). Nos levantamentos realizados em árvores recém-derrubadas no Amazonas, foram identificadas algumas espécies de besouros das famílias Scolitydae, Platypodidae, Cerambycidae e Curculionidae como causadoras de danos às toras. Esses besouros iniciam o ataque logo após o abate das árvores, permanecendo na tora até que ela não ofereça mais condições de sobrevivência (Abreu, 1989, 1992; Abreu \& Bandeira, 1992).

Esta pesquisa foi realizada com o objetivo de averiguar as condições de uso de madeiras em indústrias madeireiras de Manaus, enfocando espécies madeireiras utilizadas e medidas para evitar o ataque de insetos, como também fazer um levantamento da ocorrência de insetos em toras estocadas nos pátios das indústrias e, simultaneamente, através de observação visual, avaliar o nível de dano provocado por esses insetos.

Este trabalho foi realizado em seis indústrias madeireiras da cidade de Manaus, onde foi aplicado um questionário, objetivando identificar as espécies utilizadas, o uso de produto químico para preservar a madeira contra organismos xilófagos, o tipo de estocagem da madeiras, entre outros (Ficha anexa), o que serviu para dar subsídios à pesquisa e mostrar um pouco da realidade madeireira local. Em seguida, foi feito o levantamento da ocorrência de insetos em 19 espécies de madeira utilizadas por essas indústrias, bem como a avaliação do nível de dano causado pelas principais espécies de besouros e cupins. Pretendia-se avaliar cinco toras por espécie, porém em virtude da grande quantidade de toras e como o grau de ataque era muito grande optou-se por avaliar aquelas que se encontravam no pátio, que em muitos casos chegavam a 12 toras por espécie. O procedimento para avaliação do nível de deterioração baseou-se na observação visual das toras (casca, alburno e cerne) onde se evidenciou o ataque de coleópteros e isópteros. Em seguida determinou-se o nível de deterioração, seguindo os critérios descritos no Quadro 1, adaptados da IUFRO (1970). Para identificação da madeira e coleta dos insetos foi retirada uma amostra da tora, da região de maior infestação, abrangendo casca, alburno e cerne, e desta efetuou-se a coleta com pinça e pincel. Os insetos coletados foram conservados em álcool $70 \%$, sendo devidamente etiquetados para posterior triagem e identificação.

Os coleópteros foram identificados em nível de família, gênero e espécie, por meio da comparação direta com exemplares já anteriormente identificados e depositados na Coleção Sistemática de Invertebrados do Instituto Nacional de Pesquisas da Amazônia. Foram utilizadas, também, chaves de identificação (Anderson et al., 1996; Lawrence \& Newton, 1995; Delobel \& Tran, 1993; Wood, 1982; 1991a; 1991b; Arnett et al., 1980; Britton, 1979). Os isópteros foram identificados pelo

Quadro 1 - Classificação do nível de deterioração das toras

Table 1 - Classification of the level of deterioration of the logs

\begin{tabular}{|l|c|}
\hline \multicolumn{1}{|c|}{ Nível de Deterioração } & NOTA \\
\hline Sadio - nenhum ataque & 0 \\
\hline $\begin{array}{l}\text { Ataque leve ou superficial causado por térmitas e } \\
\text { coleópteros }\end{array}$ & 1 \\
\hline $\begin{array}{l}\text { Ataque evidente, porém moderado, causado por térmitas } \\
\text { e coleópteros }\end{array}$ & 2 \\
\hline Ataque intenso por térmitas e coleópteros & 3 \\
\hline Ataque total da casca/alburno/cerne & 4 \\
\hline
\end{tabular}


Dr. Reginaldo Constantino, da Universidade de Brasília. As madeiras coletadas foram identificadas no Laboratório de Anatomia da Madeira do CPPF/INPA.

De acordo com os resultados hdo questionário, constatou-se que as madeiras beneficiadas nas indústrias madeireiras de Manaus são oriundas das florestas de terrafirme e várzea e dos rios Purus, Negro e Solimões, distantes dos locais de beneficiamento. Sabe-se que na várzea as árvores são cortadas na época da vazante e permanecem na floresta até a época da enchente dos rios, aguardando a elevação das águas para serem transportadas. Após a derrubada, além de as toras volatilizarem substâncias atrativas para os insetos, elas apresentam um teor de umidade elevado, o que favorece o ataque de organismos xilófagos, principalmente insetos e fungos (Mendes \& Alves, 1988).

As espécies florestais beneficiadas pelas indústrias madeireiras de Manaus são, principalmente, amapá, andiroba, angelim-pedra, caucho, copaíba, cedro, jacareúba, macacarecuia, munguba, muiratinga, tintarana, seringa, sucupira, sumaúma, virola e várias espécies de louro.

Essas espécies são utilizadas para diversos fins, e geralmente o uso a que se destinam está relacionado à sua densidade. Por exemplo, assacu, macacarecuia, munguba, seringa, sumaúma e virola, espécies de baixa densidade $\left(<0,50 \mathrm{~g} / \mathrm{cm}^{3}\right)$, podem ser utilizadas na confecção de brinquedos, folhas faqueadas, compensados, laminados, formas de concreto, raquetes, pequenos objetos, cabos de vassouras, caixotaria leve, tamancos, saltos de sapatos, miolo de compensado e outros. Espécies como amapá, andiroba, angelim-pedra, cedro, copaíba, jacareúba, louro-inhamuí, louro-preto, muiratinga e tintarana, que são classificadas como de densidade média (de 0,51 a $0,72 \mathrm{~g} / \mathrm{cm}^{3}$ ), na maioria dos casos, são utilizadas na confecção de móveis em geral, laminados, obras de entalhe, compensados, assoalhos, moirões, montantes de escadas, caibros, portas, ripas, tábuas, rodapés, molduras, embalagens, folhas faqueadas decorativas, carpintaria, azimbre, marcenaria, obras internas, barris de vinho, cutelaria, cabos de instrumentos, esquadrias, vigas, pranchas, lambris etc. (Loureiro et al., 1978; 1986; INPA/CPPF, 1991, 1993; Vasconcellos, 1993).

As serrarias e fábricas de compensados/laminados pesquisadas, em sua maioria, estão instaladas próximas a cursos d'água para facilitar o acesso da matéria-prima ao processamento, onde inicialmente é feita a estocagem das toras, para depois serem içadas de dentro d'água por guindaste para o pátio em terra, sendo empilhadas umas sobre as outras, permanecendo neste local por meses, enquanto aguardam o beneficiamento, o que favorece a ação dos agentes degradadores.

Não foi possível determinar o tempo que as toras estavam estocadas dhentro d'água, pois a variação é muito grande, uma vez que muitas espécies permanecem nos pátios aquáticos das indústrias (rios e igarapés) durante vários anos. Os trabalhadores não têm controle da chegada e da retirada das toras para beneficiamento, pois isto é feito em função da procura por uma referida espécie.

De acordo com as respostas apuradas na ficha, pôdese constatar também que nenhuma das indústrias pesquisadas utilizou qualquer produto para proteger as toras contra o ataque de insetos e outros organismos xilófagos. Sabe-se que a infestação inicia-se imediatamente após a derrubada da árvore e aumenta consideravelmente, pois as toras permanecem na floresta, aguardando a elevação das águas para serem transportadas em sistema de jangadas até o local de beneficiamento. No pátio de estocagem das toras, ao ar livre, o ataque desses insetos intensifica-se, propiciado pela umidade, pela temperatura e pela existência de populações de insetos e fungos, devido à falta de profilaxia, podendo causar deterioração da madeira.

Com relação à presença de insetos nas toras, a análise dos resultados foi feita baseando-se no ataque de besouros e cupins. Nas 19 espécies madeireiras (Quadro 2) observou-se, quanto às espécies de besouros que causam danos à madeira, a predominância de Xyleborus affinis Eicchoff e Platypus parallelus Fabricius, pertencentes às famílias Scolytidae e Platypodidae, respectivamente, encontradas em 18 espécies de madeira (Figura 1), sendo conseqüentemente as maiores responsáveis pela deterioração das toras. Estas espécies são comumente encontradas em madeiras úmidas da Amazônia Central, sendo X. affinis quantitativamente maior. Em levantamentos realizados em discos de 18 espécies arbóreas, X. affinis foi encontrada em 15, dentre elas Ocotea cymbarum H.B.K. e Cedrela odorata L., enquanto $P$. parallelus foi encontrada em oito espécies (Abreu, 1989, 1992; Abreu \& Bandeira, 1992).

Outras espécies, como Xyleborus ferrugineus Fabricius, Xyleborus volvulus Fabricius, Xyleborus sp. e Platypus sp. (Quadro 2), que também pertencem às mesmas famílias, apresentaram relativa especificidade de hospedeiro, uma vez que na maioria das indústrias pesquisadas elas foram encontradas quase sempre nas

R. Árvore, Viçosa-MG, v.26, n.6, p.789-796, 2002 
Quadro 2 - Ocorrência e nível de ataque de Coleoptera e Isoptera em espécies madeireiras estocadas no pátio de indústrias madeireiras de Manaus, Amazonas

Table 2 - Occurrence and attack level of Coleoptera and Isoptera on logs stored in wood industry of Manaus, Amazonas

\begin{tabular}{|c|c|c|c|}
\hline Espécie Florestal & Família & Nível de Ataque & Inseto \\
\hline Brosimum parinarioides Ducke (amapá) & Moraceae & $1 \mathrm{CaAl}$ & $\begin{array}{l}\text { X. affinis, } \\
* \text { Staphylinidae }\end{array}$ \\
\hline Calophyllum brasiliense Cambess. (jacareúba) & Clusiaceae & $\begin{array}{l}3 \mathrm{Ca}, 2 \mathrm{Al} \\
1 \mathrm{Ca}\end{array}$ & $\begin{array}{l}\text { X. affinis } \\
\text { P. parallelus } \\
* \text { Brentidae }\end{array}$ \\
\hline Carapa guianensis Aubl. (andiroba) & Meliaceae & $\begin{array}{l}2 \mathrm{Ca}, 1 \mathrm{Al} \\
1 \mathrm{Ca}\end{array}$ & $\begin{array}{l}\text { X. affinis } \\
\text { P. parallelus } \\
\text { * Brentidae, larvas de Diptera }\end{array}$ \\
\hline Caryocar sp. (tintarana) & Caryocaraceae & $2 \mathrm{Ca} \mathrm{Al}$ & $\begin{array}{l}\text { P. parallelus } \\
\text { * Larvas de Diptera e de Coleoptera }\end{array}$ \\
\hline Cedrela odorata L. (cedro) & Meliaceae & $\begin{array}{l}3 \mathrm{Ca} \mathrm{Al}, 1 \mathrm{Ce} \\
3 \mathrm{Ca} 2 \mathrm{Al}, 1 \mathrm{Ce}\end{array}$ & $\begin{array}{l}\text { X. affinis } \\
\text { P. parallelus } \\
\text { *. Nitidulidae, larvas de Diptera, Colydiidae }\end{array}$ \\
\hline Ceiba pentandra (L.) Gaertn. (sumaúma) & Bombacaceae & $\begin{array}{l}4 \mathrm{Ca} \mathrm{Al} \\
3 \mathrm{Ca} 2 \mathrm{Al}, 1 \mathrm{Ce} \\
2 \mathrm{Ca} \mathrm{Al}, 1 \mathrm{Ce} \\
1 \mathrm{Al}\end{array}$ & $\begin{array}{l}\text { Cerambycidae } \\
X \text {. affinis } \mathrm{e} \text { P. parellelus } \\
X \text {. ferrugineus } \mathrm{e} \text {. volvulus } \\
\text { Xyleborus sp, Platypus sp e Minthea rugicolis } \\
* \text { Nitidulidae, Silvanidae, Colydiidae, Brentidae, larvas de Diptera }\end{array}$ \\
\hline Copaifera multijuga Hayne (copaíba) & $\begin{array}{l}\text { Leguminosae } \\
\text { Caesalpinioideae }\end{array}$ & $\begin{array}{l}4 \mathrm{Ca} \mathrm{Al}, 1 \mathrm{Ce} \\
4 \mathrm{Ca} \mathrm{Al} \\
3 \mathrm{Ca} \mathrm{Al}, 1 \mathrm{Ce} \\
2 \mathrm{Ca} \mathrm{Al} \\
2 \mathrm{Al} \\
1 \mathrm{Ca} \mathrm{Al}\end{array}$ & $\begin{array}{l}\text { X. affinis } \\
\text { Cerambycidae } \\
\text { P. parallelus } \\
\text { Anoplotermes sp e Platypus sp. } \\
\text { Minthea rugicolis } \\
\text { X. volvulus } \\
\text { * Scydmaenidae, Tenebrionidae, Nitidulidae, Cucujidae, Colydiidae, } \\
\text { Silvanidae, larvas de Diptera e de Coleoptera }\end{array}$ \\
\hline Copaifera sp. (copaiba) & $\begin{array}{l}\text { Leguminosae } \\
\text { Caesalpinioideae }\end{array}$ & $\begin{array}{l}2 \mathrm{Ca} \mathrm{Al}, 1 \mathrm{Ce} \\
2 \mathrm{Ca} \mathrm{AlCe} \\
2 \mathrm{Al} \\
1 \mathrm{Ca} \mathrm{Al}\end{array}$ & $\begin{array}{l}\text { X. affinis } \\
\text { P. parallelus e Platypus sp. } \\
\text { Minthea } \text { sp. } \\
\text { X. volvulus } \\
\text { * Staphylinidae, Brentidae, Histeridae, Nitidulidae }\end{array}$ \\
\hline $\begin{array}{l}\text { Couroupita guianensis Aubl. } \\
\text { (macacarecuia) }\end{array}$ & Lecythidaceae & $3 \mathrm{Ca} \mathrm{Al}, 1 \mathrm{Ce}$ & $\begin{array}{l}\text { P. paralelus X X. affinis } \\
\text { * Pselaphidae, Colydiidae }\end{array}$ \\
\hline Hevea brasiliensis Müll. Arg. (seringa) & Euphorbiaceae & $\begin{array}{l}3 \mathrm{Ca} \mathrm{Al}, 1 \mathrm{Ce} \\
2 \mathrm{Ca} \mathrm{Al}, 1 \mathrm{Ce}\end{array}$ & $\begin{array}{l}\text { P. parallelus } \\
\text { X. affinis } \\
\text { * Colydiidae, Silvanidae, Staphylinidae, larvas de Diptera }\end{array}$ \\
\hline Hura crepitans L. (açacu ou assacu) & Euphorbiaceae & $\begin{array}{l}3 \mathrm{Ca} \mathrm{Al}, 1 \mathrm{Ce} \\
2 \mathrm{Ca} \mathrm{Al}, 1 \mathrm{Ce}\end{array}$ & $\begin{array}{l}X . \text { affinis e } P \text {. parallelus } \\
\text { Platypus sp. } \\
\text { * Colydiidae, Brentidae, Nitidulidae, Staphylinidae, larvas de Diptera }\end{array}$ \\
\hline Hymenolobium sp. (angelim-pedra) & $\begin{array}{l}\text { Leguminosae } \\
\text { Papilionoideae }\end{array}$ & $\begin{array}{l}3 \mathrm{Ca} \mathrm{Al}, 1 \mathrm{Ce} \\
2 \mathrm{Ca} \mathrm{Al}\end{array}$ & $\begin{array}{l}\text { P. parallelus e } X \text {. affinis } \\
X \text {. volvulus e Cerambycidae } \\
\text { Tenebrionidae, Colydiidae, Cucujidae } \\
\text { * Elateridae, Cucujidae, larvas de Diptera }\end{array}$ \\
\hline $\begin{array}{l}\text { Maquira coriacea (Karsten) C. C. Berg } \\
\text { (muiratinga) }\end{array}$ & Moraceae & $\begin{array}{l}3 \mathrm{Ca} \mathrm{Al}, 1 \mathrm{Ce} \\
2 \mathrm{Al}\end{array}$ & $\begin{array}{l}X \text {. affinis e } P \text {. parallelus } \\
\text { Minthea } \mathrm{sp} \text {. } \\
\text { * Nitidulidae, Staphylinidae }\end{array}$ \\
\hline Micranda sp. (caucho) & Euphorbiaceae & $\begin{array}{l}2 \mathrm{Ca} \mathrm{Al} \\
1 \mathrm{Ca} \mathrm{Al}, 3 \mathrm{Ce}\end{array}$ & $\begin{array}{l}\text { P. parallelus, } X \text {. affinis e Cerambycidae } \\
\text { Nasutitermes corniger } \\
\text { * Colydiidae, Nitidulidae, Staphylinidae, larvas de Coleoptera }\end{array}$ \\
\hline Nectandra sp.(louro-preto) & Lauraceae & $3 \mathrm{Ca} \mathrm{Al}, 2 \mathrm{Ce}$ & X. affinis e P.parallelus \\
\hline Ocotea cymbarum H.B.K. (Louro-inhamuí) & Lauraceae & $2 \mathrm{CaAl}$ & X. affinis e $P$. parallelus \\
\hline $\begin{array}{l}\text { Pseudobombax munguba (Mart. et Zucc.) A. } \\
\text { Robyns (Munguba) }\end{array}$ & Bombacaceae & $2 \mathrm{Ca} \mathrm{Al}, 1 \mathrm{Ce}$ & P. parallelus e X affinis \\
\hline Virola surinamensis Warb. (virola) & Myristicaceae & $\begin{array}{l}3 \mathrm{Ca} \mathrm{Al}, 1 \mathrm{Ce} \\
3 \mathrm{Ce} \\
2 \mathrm{Ca} \mathrm{Al} \\
2 \mathrm{Ca}, 1 \mathrm{Al} \\
1 \mathrm{Ca} \mathrm{Al}\end{array}$ & $\begin{array}{l}\text { X. affinis e } P \text {. parallelus } \\
\text { Nasutitermes corniger } \\
\text { Cerambycidae } \\
\text { X. ferrugineus e Xyleborus sp. } \\
X \text {. volvulus, Platypus sp., Cnesinus sp. e Dinoderus bifoveolatus } \\
* \text { Silvanidae, Staphylinidae, larvas de Diptera, Colydiidae, Cucujidae, } \\
\text { Nitidulidae }\end{array}$ \\
\hline Vochysia sp. (louro-preto) & Vochysiaceae & $\begin{array}{l}3 \mathrm{Ca} \mathrm{Al}, 1 \mathrm{Ce} \\
2 \mathrm{Ca}, 1 \mathrm{Al}\end{array}$ & $\begin{array}{l}\text { X. affinis e Xyleborus sp. } \\
\text { X. ferrugineus, } P \text {. parallelus e Platypus sp. } \\
* \text { Larvas de Diptera }\end{array}$ \\
\hline
\end{tabular}

1 - ataque superficial, 2 - ataque moderado, 3 - ataque intenso e 4 - ataque total; $\mathrm{Ca}$ - Casca, Al - Alburno e Ce - Cerne.

* Insetos não relacionados à deterioração das madeiras. 
mesmas espécies e o ataque, em grande parte das toras, variou de superficial (nota 1) a moderado (nota 2). A despeito de $X$. ferrugineus ter tido baixa ocorrência nas madeiras (Figura 1), ela é uma das espécies mais abundantes nos trópicos das Américas e do leste da Índia (Saunders et al., 1967). Em trabalhos realizados por Abreu (1992) e por Abreu \& Bandeira (1992), também foi observado que a infestação desta espécie em madeiras da Amazônia é considerada de baixa intensidade.

Os insetos mencionados fazem parte do grupo de besouros que se alimentam de fungos por eles cultivados dentro das galerias das madeiras, possuindo, assim, o hábito de atacar somente árvores decadentes, doentes e recém-derrubadas, onde ocorre a fermentação da madeira, originando substâncias que servem como atrativo. Eles preferem ainda substratos com altos teores de umidade (Gagne \& Kearby, 1978; Moeck, 1981). Segundo Gray (1972), é quase impossível uma árvore recém-derrubada não ser atacada por insetos das famílias Scolytidae e Platypodidae, a não ser que receba um produto preservante imediatamente após o abate.

Todas as partes da tora foram atacadas por esses besouros, mas as regiões com maior concentração de ataque foram a casca e o alburno (Quadro 2), onde o nível de infestação em muitas espécies atingiu a nota 4. Sabese que nessas regiões há maior concentração de nutrientes, principalmente proteínas e vitaminas, que são importantes para o desenvolvimento de fungos e, conseqüentemente, passam para os insetos, sendo incorporados através da cadeia alimentar (Batra, 1963). A quantificação dos danos provocados por estes insetos em espécies comerciais ainda é muito pequena, principalmente levando-se em consideração os orifícios e as galerias na casca e no alburno, porém quando são avaliadas as manchas ocasionadas pelos fungos que se estendem além das linhas das galerias o valor da madeira é depreciado. Segundo Mclean \& Borden (1977), o efeito da infestação do fungo na madeira resulta em mancha de cor escura, quase negra, que vai além das linhas das galerias, penetrando no interior da madeira. Por causa destas manchas e das galerias, a parte do alburno das madeiras nas indústrias pesquisadas, em sua maioria, é descartada no momento do processamento das toras. Quando o ataque adentra o cerne, como foi observado em várias espécies, torna-se mais difícil o aproveitamento da madeira, aumentando assim o prejuízo. Outro fator que contribui para o agravamento deste quadro é que a tora demora a ser processada, o que permite que os besouros completem um ciclo de vida e comecem novas gerações.

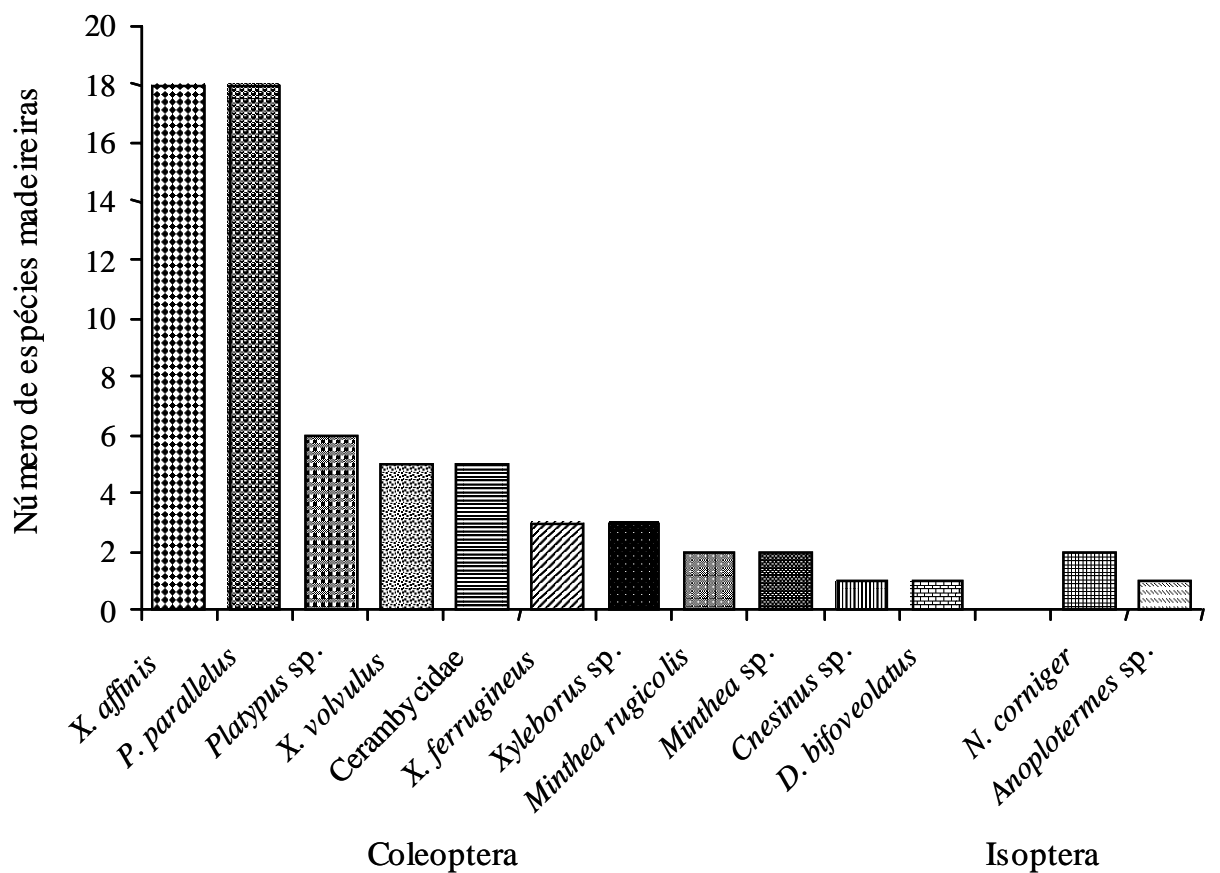

Figura 1 - Número de espécies madeireiras atacadas por insetos das ordens Coleoptera e Isoptera. Figure 1 - Number of wood species attacked by insect of Coleoptera and Isoptera orders. 
O conteúdo de umidade da madeira é provavelmente o fator físico mais importante que afeta as condições de ataque dos besouros-ambrosia, não somente por causa do gradiente de umidade, mas também devido à pressão osmótica que pode ser responsável ou estar relacionada com a produção de substâncias atrativas (Danthanarayana, 1973; Bletchly, 1961). Segundo Reid (1963), citado por Danthanarayana (1973), esse também pode ser um fator limitante para o desenvolvimento do fungo, uma vez que a umidade abaixo de $50 \%$ limita seu crescimento e, conseqüentemente, reduz a alimentação dos besouros. Segundo Deón (1989), a alta umidade é condição básica para o desenvolvimento de besouros xilomicetófagos em madeira verde. Quando a umidade diminui os besouros deixam a madeira em busca de outro substrato, devido à morte dos fungos que servem de alimento.

Das 19 espécies de madeiras estudadas, cabe salientar que apenas Brosimum parinarioides (amapá) não foi atacada por Platypodidae, assim como Caryocar sp. (tintarana) não sofreu ataque de Scolytidae (Quadro 2).

As espécies Minthea rugicolis Walk, Minthea sp. (Lyctidae) e Dinoderus bifoveolatus Wollaston (Bostrichidae) pertencem ao grupo de insetos que se alimentam de amido (Bletchly, 1967; Furnis \& Carolin, 1977). O número de espécies de madeira atacadas por esses insetos foi baixo (Figura 1). Observou-se também que elas foram encontradas somente naquelas madeiras utilizadas por indústrias de compensado e que o grau de ataque foi considerado moderado (Quadro 2). M. rugicolis é largamente distribuída nas regiões tropicais, sendo considerada uma das principais pragas de madeira seca e de móveis (Gray, 1972). Dinoderus bifoveolatus, apesar de preferencialmente se alimentar, tanto na fase adulta como na fase larval, de amido de madeira seca, também ataca outros tipos de substrato, como macaxeira processada (Schafer et al., 2000).

A família Cerambycidae, cujas larvas foram encontradas atacando principalmente a casca e o alburno de Copaifera multijuga (copaíba), Ceiba pentandra (sumaúma), Micranda sp. (caucho) e Pseudobombax munguba (munguba), espécies em sua maioria utilizadas pelas indústrias de compensado (Quadro 2), é uma importante praga florestal. Segundo Hickin \& Edwards (1975), as larvas constroem galerias na casca, no alburno, no cerne, nas raízes, nas árvores vivas e mortas etc.

R. Árvore, Viçosa-MG, v.26, n.6, p.789-796, 2002
As demais famílias de Coleoptera encontrhadas (Quadro 2) são consideradas importantes do ponto de vista ecológico, pois participam da cadeia alimentar, sendo predadoras de outros artrópodos. Algumas espécies são encontradas vivendo em galerias de besouros de casca e brocas de madeiras, alimentando-se de suas larvas. Como exemplo têm-se Staphylinidae, Colydiidae, Silvanidae Cucujidae e Histeridae, que são predadoras de Scolytidae e Platypodidae (Wood, 1982; Furnis \& Carolin, 1977). Outros casos são os Nitidulidae, que também se alimentam da seiva fermentada, debaixo da casca (Borror et al., 1992).

Foram observadas somente duas espécies de cupins atacando as espécies estudadas: Nasutitermes corniger (Motschulsky) e Anoplotermes sp. (Quadro 2). $N$. corniger teve preferência por espécies de madeiras utilizadas em indústrias de compensado e pela região do cerne, que apresenta alta concentração de celulose e hemicelulose, fontes de alimentos desses insetos. Segundo Bandeira et al. (1989), este inseto destrói madeira dura ou mole, seca ou úmida, beneficiada ou não. $\mathrm{Na}$ Amazônia brasileira ela é encontrada no Estado do Amazonas (Constantino \& Cancelo, 1992), e é uma das espécies mais comuns e de maior extensão geográfica na região neotropical (Araújo, 1970). Apesar de Anoplotermes sp. ter sido encontrada atacando a casca e o alburno, de acordo com Bandeira (1989), ela se alimenta de húmus.

Deste trabalho pode-se concluir que, das seis indústrias pesquisadas, nenhuma delas utiliza produtos químicos contra insetos xilófagos e que a secagem e a estocagem das toras são feitas de forma incorreta, sendo elas empilhadas umas sobre as outras.

Foram capturadas 11 espécies de Coleoptera e duas de Isoptera, tendo as espécies Xyleborus affinis e Platypus paralelus sido encontradas em 18 espécies florestais analisadas, portanto são as responsáveis pela maioria dos danos nas toras. Constatou-se que as regiões das toras com maior intensidade de ataque foram a casca e o alburno.

\section{AGRADECIMENTO}

Aos técnicos Alfredo Pereira dos Santos, Frank Antônio de Oliveira Campos e Orleilson Morais de Lima, pela coleta e triagem dos insetos. 


\section{FICHA PARA PREENCHIMENTO NAS EMPRESAS MADEIREIRAS}

01. Nome da Indústria:

02. Espécie de madeira que trabalham

Nome comum:

03. Região de origem da madeira:

04. Qual o tempo aproximado desde o corte até a estocagem das toras?
( ) horas
( ) dias
( ) meses

05. Tipo de estocagem da tora:
( ) em pátio ao ar livre
( ) imersa no rio
( ) em contato com o solo
( ) empilhada com o uso de separadores
( ) empilhada sem o uso de separadores

06. Umidade da madeira:
( ) verde
( ) seca ao ar livre
( ) seca em estufa

07. A tora/tábua sofreu algum tratamento preventivo?
( ) $\mathrm{Sim}$
( ) Não

Qual o produto preservante?

Qual o tipo de tratamento?

08. Espécies de insetos encontrados:

Ordem:

Família:

Espécie:

OBS.:

\section{REFERÊNCIAS BIBLIOGRÁFICAS}

ABREU, R. L. S.; DIETRICH, C. R. R. C. Ocorrência de besouros (Insecta: Coleoptera) em madeiras úmidas. In: ENCONTRO BRASILEIRO DE MADEIRA E EM ESTRUTURAS DE MADEIRA, 3., 1989, São Carlos. Anais... São Carlos: 1989. p. 227-237,

ABREU, R. L. S. Estudo da ocorrência de Scolytidae e Platypodidae em madeiras da Amazônia. Acta Amazonica, v. 22, n. 3, p. 413-420, 1992.
ABREU, R. L. S.; BANDEIRA, A. G. Besouros xilomicetófagos economicamente importantes da região de Balbina, Estado do Amazonas. Revista Árvore, v. 16, n. 3, p. 346-356, 1992.

ANDERSON, D. M. et al. Chaves Ilustradas para coleópteros adultos e larvas (Coleoptera) encontrados associados com madeiras em portos de entrada. Tradução Amauri M. J. Rodrigues. Brasília: MA/SDA/DDIV. 1996. $24 \mathrm{p}$.

ARNETT JR., R. H.; DOWNIE, N. M.; JAQUES, H. E. How to know the beetles. 2.ed. Dubuque: Brown Company Publishers, 1980. $416 \mathrm{p}$.

ARAÚJO, R. Termites of the neotropical region. In: KRISNA, K.; WEESNER, F. (Eds.). Biology of Termites. London: Academic Press, 1970. p. 527-576.

BANDEIRA, A. G. Análise da termitofauna (Insecta: Isoptera) de uma floresta primária e de uma pastagem na Amazônia Oriental, Brasil. Boletim do Museu Paraense Emílio Goeldi, sér. Zool., v. 5, n. 2, p. 225-241, 1989.

BANDEIRA, A. G. et al. Insetos pragas de madeiras de edificações em Belém - Pará. Belém: EMBRAPA/CPATU, 1989. 24 p. (Boletim de Pesquisa, 101)

BATRA, L. R. Ecology of ambrosia fungi and their dissemination by beetles. Transactions of the Kansas Academy of Science, v. 66, n. 2, p. 213-236, 1963.

BORROR, D. J.; TRIPLEHORN, C. H.; JOHNSON, N. F. An introduction to the study of the insects. 6.ed. Orlando, Florida: Saunders College Publishing, 1992. $780 \mathrm{p}$.

BRITTON, E. B. Coleoptera. In: The insects of Australia. Canberra: Melbourne University Press. 1979. p. $495-621$.

BLETCHLY, J. D. Insect and marine borer damage to timber and woodwork. Recognition, prevention and eradication. London: Her Majesty's Stationery Office, 1967. $88 \mathrm{p}$.

BLETCHLY, J. D. A review of factors affecting ambrosia beetles attack in trees and felled logs. Empire Forestry Review, v. 40, n. 1, p. 13-18, 1961.

CONSTANTINO, R.; CANCELLO, E. M. Cupins (Insecta, Isoptera) da Amazônia Brasileira: Distribuição geográfica e esforço de coleta. Revista Brasileira de Biologia, v. 52, n. 3, p. 401-413, 1992.

R. Árvore, Viçosa-MG, v.26, n.6, p.789-796, 2002 
DANTHANARAYANA, W. Host plant-pest relationships of the shot-hole borer of tea (Xyleborus fornicatus) (Coleoptera: Scolytidae). Entomologia Experimental \& Applicatta, v. 16, p. 305-312, 1973.

DELOBEL, A. ; TRAN, M. Les Coléoptères des denrées alimentaires antreposées dans les régions chaudes. Paris: Orstom., 1993. 410 p.

DÉON, G. Manual de preservação das madeiras em clima tropical. Trad. Antônio C. Mascarenhas. Salvador: Escola Politécnica da Universidade Federal da Bahia. 1989. 116p. (Série Técnica, 3)

FURNIS, R. L.; CAROLIN, V. M. Western forest insects. Washington: USDA, 1977. 654 p. (Miscellaneous Publication, 1339)

GRAY, B. Economic tropical forest entomology. Annual Review Entomology, v. 17, p. 313-335, 1972.

GAGNE, J. A.; KEARBY, W. H. Host selection by Xyleborus celsus (Coleoptera: Scolytidae) in Missouri. Canadian Entomologist, v. 110, n. 10, p. 1009-1013, 1978.

HICKIN, N. E.; EDWARDS, R. The insect factor in wood decay. London: Associated Business Programmes London, 1975. $383 \mathrm{p}$.

INSTITUTO NACIONAL DE PESQUISA DA AMAZÔNIA - INPA/CPPF. Catálogo de madeiras da Amazônia: Características tecnológicas. Manaus: 1991. 163 p.

INSTITUTO NACIONAL DE PESQUISA DA AMAZÔNIA - INPA/CPPF. Catálogo de madeiras do Amapá:

Características tecnológicas. Manaus: 1993. 58 p.

IUFRO. Método padrão sugerido pela IUFRO para ensaios de campo com estacas de madeira.Trad. Lepage. E.S.

Preservação de Madeira, v. 1, n. 4, p. 205-216, 1970.

LAWRENCE, J. F.; NEWTON, JR., A. F. Families and subfamilies of coleoptera (with selected genera, notes, references and date on family-group names). Warszawa: Muzeum i Instytut Zoologii PAN, 1995. 1006 p.

LOUREIRO, A. A.; VASCONCELLOS, F. J.; FREITAS, J. A. Anatomia do lenho de cinco espécies e duas variedades de Diplotropis (Fabaceae) da Amazônia. Acta Forestalia Brasiliensis, v. 1, n. 1, p. 19-42, 1986.

LOUREIRO, A. A.; SILVA, M. F.; ALENCAR, J. C. Essências madeireiras da Amazônia. Manaus: Suframa, 1978. $2 \mathrm{v}$.
MCLEAN, J. A.; BORDEN, J. H. Attack by Gnathotrichus sulcatus (Coleoptera: Scolytidae) on stumps and felled trees baited with sulcatol and ethanol. Canadian Entomologist, v. 109 , p. $675-686,1977$.

MENDES, A. S.; ALVES, M. V. S. A degradação da madeira e sua preservação. Brasília: Instituto Brasileiro do Desenvolvimento Florestal/Laboratório de Produtos Florestais, 1988. 57 p.

MOECK, H. A. Ethanol induces attack on trees by spruce beetles, Dendroctonus rufipennis (Coleoptera: Scolytidae). Canadian Entomologist, v. 113, p. 939-942, 1981.

SALES-CAMPOS, C.; ABREU, R. L. S.; VIANEZ, B. F. Condições de uso e processamento de madeira nas indústrias madeireiras de Manaus, Amazonas, Brasil. Acta Amazonica, v. 30, n. 2, p. 319-331, 2000.

SAUNDER, J. L.; NORRIS, D. M.; KNOKE, J. K. Insecthost tissue interrelations between Xyleborus ferrugineus (Coleoptera: Scolytidae) and Theobroma cacao in Costa Rica. Annals of Entomological Society of America, v. 60, n. 2, p. 419-423, 1967.

SCHAFER, K.; GEORGEN, G.; BORGEMEISTER, C. An illustrated identification key to four different species of adult Dinoderus (Coleoptera: Bostrichidae), comment attacking dried cassava chips in West Africa. Journal of Stored Products Research, v. 36, p. 245-252, 2000.

VASCONCELLOS, F. J.; SILVA, A. C. E.; FREITAS, J. A. Deposição de sílica e cristais no xilema de espécies tropicais da família Caesalpinaceae. Revista Árvore, v. 17, n. 3, p. 369-374, 1993.

WOOD, S. L. Los Scolytidae de Costa Rica: clave de géneros y de la subfamilia Hylesinae (Coleoptera). Revista de Biologia Tropical, v. 39, n. 1, p. 125-148, 1991a.

WOOD, S. L. Scolytidae (Coleoptera) de Costa Rica II. Clave para la subfamilia Scolytinae, tribus: Scolytini, Ctenophorini, Micracini, Ipini, Dryocoetini, Xyleborini y Cryphalini. Revista de Biologia Tropical, v. 39, n. 2, p. 279-306, 1991 b.

WOOD, S. L. The bark and ambrosia beetles of North and Central America (Coleoptera: Scolytidae), a taxonomic monograph. Provo: 1982. 1360 p. (The Great Basin Naturalist Memoirs, 6) 